\title{
RECONN: A CYTOSCAPE PLUG-IN FOR EXPLORING AND VISUALIZING REACTOME
}

\author{
WILLEM P.A. LIGTENBERG*, DRAGAN BOŠNAČKI \\ and PETER A. J. HILBERS \\ BioModeling and BioInformatics, Department of BioMedical \\ Engineering, Eindhoven University of Technology \\ Eindhoven, $5600 \mathrm{MB}$, The Netherlands \\ *W.P.A.Ligtenberg@tue.nl
}

Received 12 April 2012

Revised 24 December 2012

Accepted 26 December 2012

Published 18 March 2013

\begin{abstract}
Interface and visualization tools usually provide static representations of biological pathways, which can be a severe limitation: fixed pathway boundaries are used without consensus about the elements that should be included in a particular pathway; one cannot generate new pathways or produce selective views of existing pathways. Also, the tools are not capable of integrating multiple levels that conceptually can be distinguished in biological systems.

We present ReConn, an interface and visualization tool for a flexible analysis of large data at multiple biological levels. ReConn (Reactome Connector) is an open source extension to Cytoscape which allows user friendly interaction with the Reactome database. ReConn can use both predefined Reactome pathways as well as generate new pathways. A pathway can be derived by starting from any given metabolite and existing pathways can be extended by adding related reactions. The tool can also retrieve alternative routes between elements of a biological network. Such an option is potentially applicable in the design and analysis of knockout experiments. ReConn displays information about multiple levels of the system in one view. With these dynamic features ReConn addresses all of the above mentioned limitations of the interface tools.
\end{abstract}

Keywords: Cytoscape; Reactome; interactive visualization; biological networks; multiscale; pathways; dynamic.

\section{Introduction}

In bioinformatics research a plethora of interface and visualization tools have been developed to cope with the increasing amounts of data in the context of different omics. ${ }^{1-5}$ Large data sets are produced by high-throughput experiments and are available from the numerous data bases. ${ }^{6-12}$ Biological networks and in particular, various types of pathways, play a central role in the organization and representation

${ }^{*}$ Corresponding author. 
of the data. The pathways are usually represented in a static manner, which can incur significant limitations. For instance, pathways are represented within fixed boundaries. Since in many cases the choice which element to assign to a pathway can be quite subjective, the pathways differ depending on the data source. Moreover, the tools lack the capability to dynamically construct pathways based on some predefined conditions. Also, one often cannot select parts of the pathways, i.e. one is restricted to displaying the whole pathway, which can make the analysis and interpretation of the data more difficult.

In biological system different scales are distinguished that interact with each other. Genes, proteins, cells, organs, each can be viewed as a separate level with its own structure and dynamics. Usually, measurement techniques gather data at a single level. Examples on the gene level include microarrays ${ }^{13}$ and SAGE, ${ }^{14}$ and on the protein level western blot ${ }^{15}$ and high-throughput mass spectrometry. ${ }^{16}$ Analysis of the pathways can be done on a single level (e.g. genes or proteins) as well as on multiple levels (e.g. the combination of genetic and protein data). Each level may be considered as a network of interactions. To find patterns that span multiple levels, these networks need to be combined. Therefore, the challenge is to integrate data across different levels and scales into one single network. Unfortunately, the interface and visualization tools often lack the capability of dealing with multiple levels in a satisfactory manner.

Reactome $^{6}$ is a publicly available open source database of biological pathways. It contains data sets for several organisms, the human one being the largest. In Reactome the basic elements are reactions (also called steps) and they are grouped in pathways. Cytoscape ${ }^{17}$ is an open source software for visualizing biological networks and integrating them with experimental data. Additional features can be added to Cytoscape as plug-ins.

In this paper we present ReConn (Reactome Connector), a Cytoscape plug-in that interacts with the Reactome database. In ReConn a pathway can be derived by starting from any given metabolite and existing pathways can be extended by adding related reactions. The tool can retrieve alternative routes between elements of the network. This option is potentially applicable in the design and analysis of knockout experiments. In this paper we describe the capabilities that ReConn provides from a user point of view and also give some highlights of the implementation of the main features.

Interface and visualization software needs to meet several basic requirements. We make an inventory of these important features and perform a comparative analysis of some existing tools, including ReConn. As a result we conclude that no tool is complete with regard to the requirements. However, we show that ReConn has better coverage of these features than the other tools.

\section{ReConn Features}

Apart from visualization of pathway information from the Reactome database, with ReConn users also can map their own data on pathways and query the data in ways 
which are not provided by other interface and visualization tools. With the clientserver approach employed in ReConn users need not keep their pathway files up to date, which eliminates the overhead by the download of large data sets. These and other new features that ReConn adds to Cytoscape are discussed in more detail in this and the subsequent sections. We begin with an overview of the organization of the Reactome database and the pathway data in particular, that is retrieved by ReConn.

Although Reactome is a curated and a peer-reviewed resource of human biological processes, it also contains data from other species. Most of the reactions in other species are computationally derived from their human counterpart, although there are some species specific reactions in Reactome. As the name "Reactome" suggests, the most important entity in the database is a reaction. Reactions are grouped in pathways and pathways are themselves connected in a hierarchical manner. The Reactome data model allows for a large variety of processes to be stored because a reaction is not limited to a metabolic reaction. For example, transport processes are also considered a reaction, since they "transform" a metabolite from a certain compartment of the cell into the same metabolite in another compartment of the cell.

\subsection{Retrieving pathway information}

ReConn has several options for querying Reactome for pathway information and visualization. One can search the database on (a part of) the name of the pathway. Another possibility is to specify a metabolite from which to start to "grow" a pathway. Also, it is possible to visualize all reactions on screen. For each of these options, it is possible to specify the species, either by selecting them from the list or by specifying the taxon identifier.

Figure 1 gives an example of the visualization of the Pyruvate metabolism and the TCA cycle for Homo sapiens in ReConn. This picture has been obtained by searching for the term "TCA" and subsequently expanding the pathway nodes. ReConn uses various different elements on the screen, which are explained in the inset.

For "growing" a pathway, ReConn uses a breadth-first search algorithm. The first step in the algorithm is to select a starting node. Then it adds the nodes that are connected to the current selection. This continues until the selection does not change any more. The starting node (source) is given by the set of reactions that have a user-specified metabolite as a substrate. For this algorithm, we have used the links between reactions as specified by Reactome. With the precedingEvent attribute, from the Reactome data model, Reactome specifies which event precedes a given event. Events can be a pathway, a reaction, or a so called black-box event.

\subsection{Calculating alternative routes}

In knockout experiments some reaction nodes of the original network are disabled and as a result reaction chains can be broken. Thus, in the design and analysis of such experiments it could be beneficial to know whether two given nodes remain 


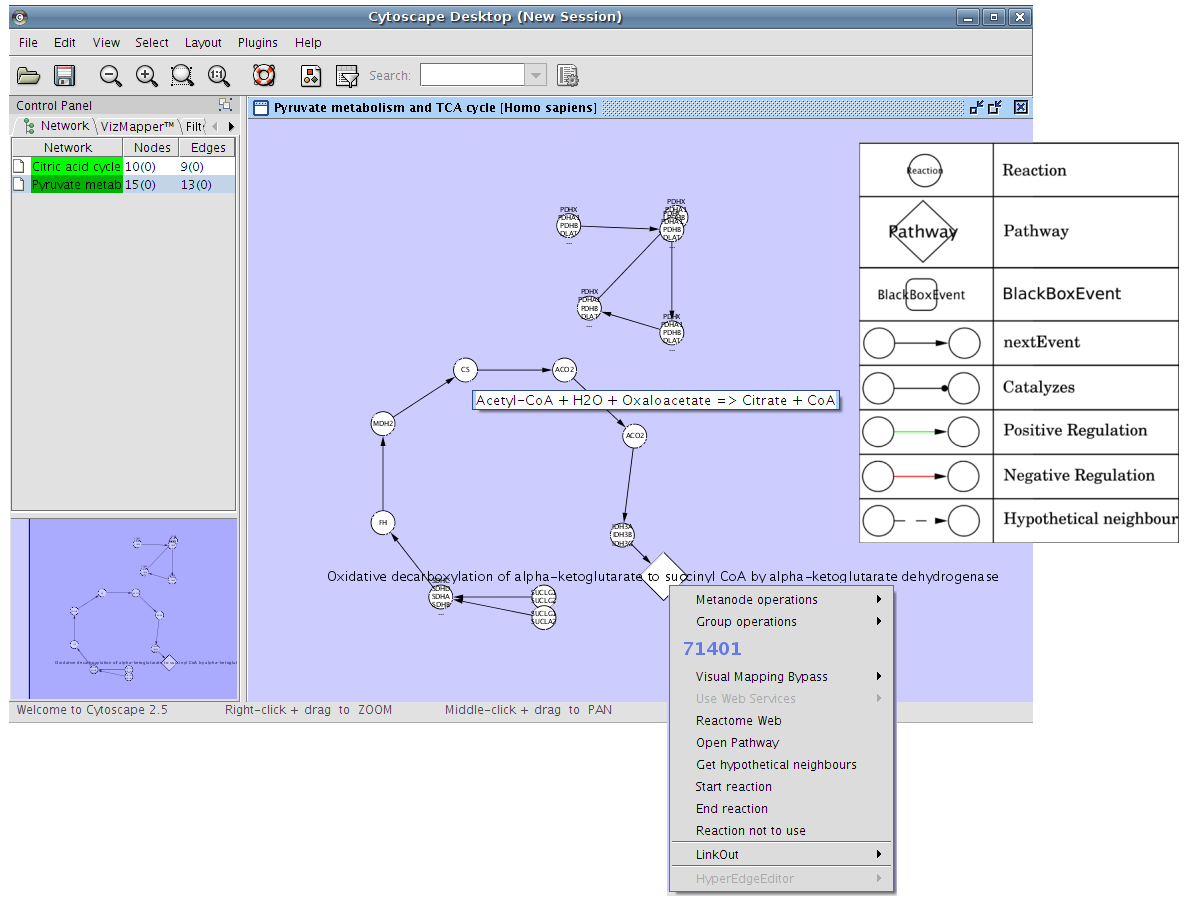

Fig. 1. The Pyruvate metabolism and TCA cycle in ReConn. This screen shot shows the Pyruvate metabolism and TCA cycle. This picture has been obtained by searching for the term "TCA" and afterwards expanding the nodes. The different visual elements are explained in the legend. The figure also shows the context menu.

connected after some nodes are removed from the pathway. For that purpose, a feature is required which calculates alternative routes. This feature can also be applied to experiments in which more than one reaction is knocked out. By using the context menu the user can specify the start reaction, end reaction, and the reactions to exclude. After the selection, the process of route calculation can be started from the ReConn menu. The graph is then generated with a breadth-first search, beginning from the given start reaction. From each reaction (node) we consider all edges defined with the precedingEvent attribute. Besides that we assume that there is an edge between reactions $r_{1}$ and $r_{2}$, if the output of $r_{1}$ is used as input for $r_{2}$ and $r_{1}$ and $r_{2}$ belong to the same compartment (see also the section "Retrieving hypothetical neighbors" below). Reactions that have been excluded by the user are not used in the breadth-first search. After this step, a backwards breadth-first search is performed starting from the end reaction. This removes reactions that are not in any path from the start to the end node.

A pseudo code of the algorithm is given in Algorithm 1. An example of how the algorithm works is illustrated in Fig. 2. The source graph gives the restricted network which does not contain the nodes excluded by the user and which is the input for the algorithm. The start and end nodes are given in green and red, respectively. In step 1 

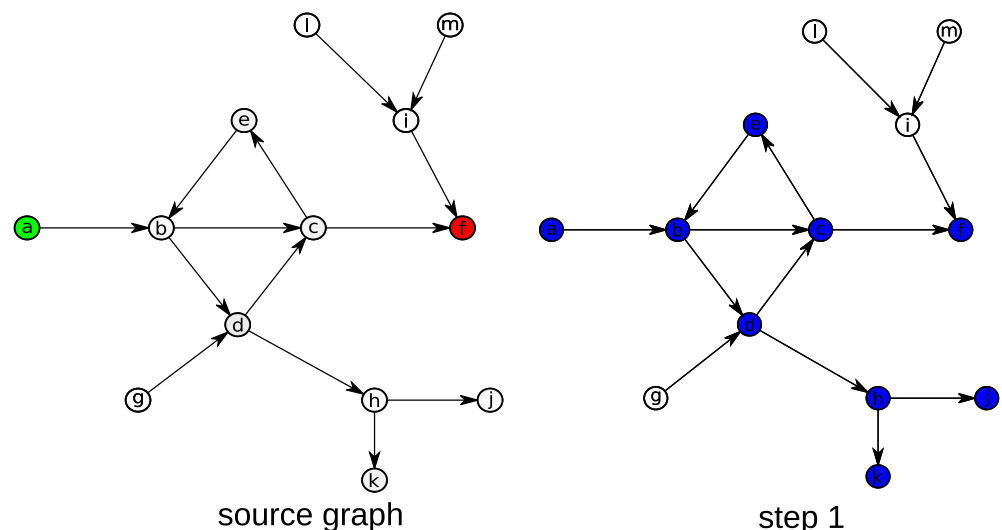

step 1

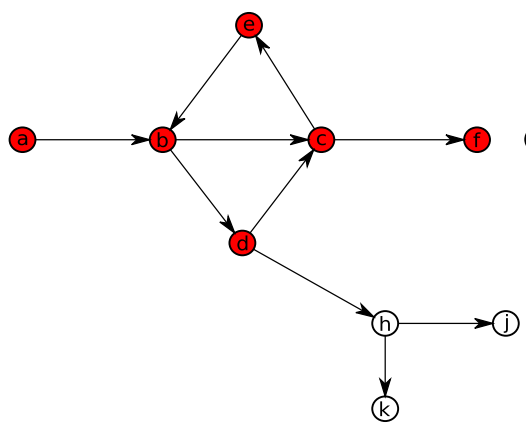

step 2

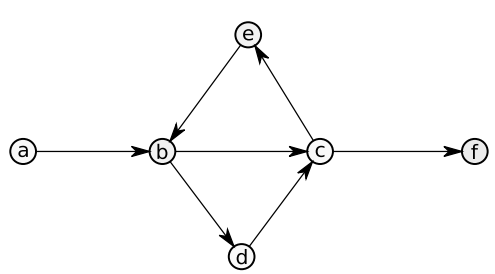

result

Fig. 2. Illustration of the Alternative Pathways algorithm. An illustration in which the various steps of the Alternative Pathways algorithm are depicted.

\subsubsection{Visualization of experimental data}

Experimental data should be put into context in order to interpret it. To facilitate this, ReConn can import data and visualize it onto the pathway that is on screen. The data can even be visualized on the overview of all reactions, which contains over 1900 reactions.

\subsubsection{Finding pathways by data}

Another application is that of finding the pathways that are influenced by an experiment. For example, when a drug is tested on single cells, one might be interested in finding unwanted effects of the drug in other pathways. In such cases, the PathwayFinder option from the ReConn menu can be used. ReConn will then map all the identifiers of a specified data file to reactions in Reactome. All these reactions belong to one or more pathways. These pathways are shown as a tree structure in a separate window (see Fig. 3). A tree structure was chosen, since pathways are ordered hierarchically in the Reactome database. 


\begin{tabular}{|c|c|c|c|}
\hline$\underline{\underline{\underline{9}}}$ & PathwayFinder & $\square$ & \\
\hline \multicolumn{4}{|c|}{ Pathways } \\
\hline \multicolumn{4}{|c|}{ Breakdown of hydrogen peroxide to water and } \\
\hline \multicolumn{4}{|c|}{ Membrane Trafficking [Homo sapiens] $(9 / 14)$} \\
\hline \multicolumn{4}{|c|}{$+\square \mathrm{M}$} \\
\hline \multicolumn{4}{|c|}{$+\cdots$ Apoptosis [Homo sapiens] $(75 / 15$} \\
\hline \multicolumn{4}{|c|}{ Electron Transport Chain [Homo sapiens] $(6 / 6)$} \\
\hline \\
\hline \multirow{2}{*}{\multicolumn{4}{|c|}{$\begin{array}{l}\text { +.. Mitochondrial Uncoupling Proteins [Hon } \\
+\square \text { Hemostasis [Homo sapiens] (49/262) }\end{array}$}} \\
\hline & & & \\
\hline \multicolumn{4}{|c|}{$+\square$ DNA Replication [Homo sapiens] (61/103) } \\
\hline \multirow{2}{*}{\multicolumn{4}{|c|}{$\begin{array}{l}+\cdots 3^{\prime} \text {-UTR-mediated translational regulation [Homo } \\
+\cdots \square \text { Cell Cvcle Checkpoints [Homo sapiens] }(37 / 59)\end{array}$}} \\
\hline & Cell Cycle Checkpoints [Homo sapie & & \\
\hline \multicolumn{4}{|c|}{ VA Repair [Homo sapiens] $(118 / 741)$} \\
\hline \multicolumn{4}{|c|}{..$-[$ Signal attenuation [Homo sapiens] $(6 / 6)$} \\
\hline \multicolumn{4}{|c|}{$\square$ Butyryl-ACP biosynthesis [Homo sapiens] (3/9) } \\
\hline \multicolumn{4}{|c|}{$\square$ Telomere Maintenance [Homo sapiens] $(23 / 43)$} \\
\hline \multicolumn{4}{|c|}{ [ Pausing and recovery of Tat-mediated HIV-1 elongation $[\mathrm{H}$} \\
\hline \multicolumn{4}{|c|}{$\square$ Lipid and lipoprotein metabolism [Homo sapiens] (115/56 } \\
\hline \multicolumn{4}{|c|}{ Transcription [Homo sapiens] $(87 / 113)$} \\
\hline \multicolumn{4}{|c|}{$\square$ Metabolism of xenobiotics [Homo sapiens] $(37 / 143)$} \\
\hline \multirow{12}{*}{\multicolumn{4}{|c|}{ 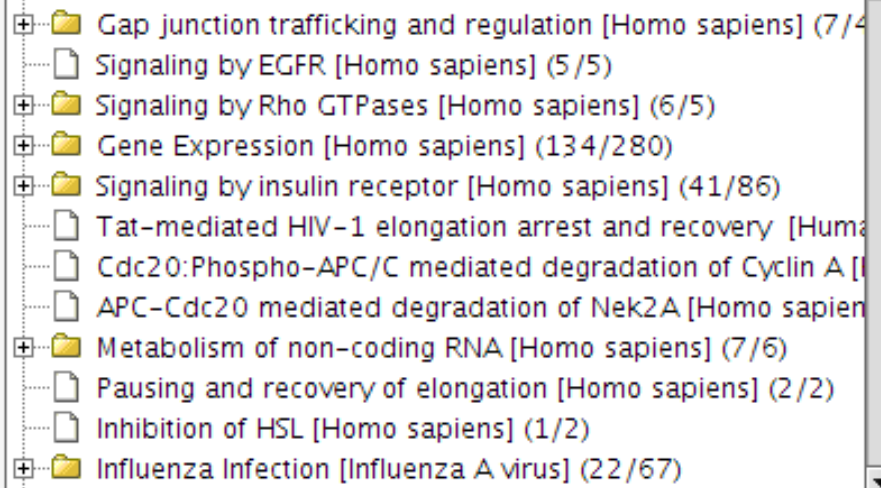 }} \\
\hline & & & \\
\hline & & & \\
\hline & & & \\
\hline & & & \\
\hline & & & \\
\hline & & & \\
\hline & & & \\
\hline & & & \\
\hline & & & \\
\hline & & & \\
\hline & & & \\
\hline \multicolumn{3}{|c|}{ 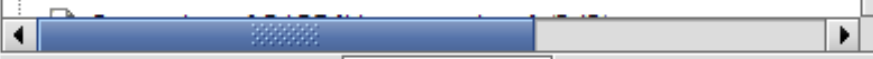 } & \\
\hline & & & \\
\hline
\end{tabular}

Fig. 3. The tree structure of pathways. The tree structure generated by PathwayFinder based upon the Reactome's organization of pathways.

\subsubsection{Coloring of nodes by occurrence}

Many experiments are performed on model organisms, which can differ from humans. ReConn has the option to color the reactions according to how widely they are used in the various organisms. The lighter the reaction is, the less widespread its use. This could prove useful if the results of an experiment a model organism are to be translated to other organisms. 


\subsubsection{Linking out to Reactome}

If at any time the user requires more information on a reaction, he or she can right-click on a node to open the context menu and click on Reactome Web to go to the Reactome website. The website will open with the information about the clicked object. The context menu is also shown in Fig. 1. This is another way to economize in the interest of clarity with the amount of information which is stored and/or shown in the visualization. Thus, not all data needs to be stored locally. Instead, extra information can be acquired on demand by browsing the Reactome web site.

\subsubsection{Expanding pathway nodes}

Pathway nodes can be expanded to see which reactions are part of the pathway. This can be achieved by selecting "Open Pathway" from the context menu (Fig. 1). This will add the reactions from the pathway to the current network. By using pathway nodes instead of only reaction nodes allows to represent the top pathway of the hierarchical structure of Reactome. Moreover, this can be done without immediately representing hundreds of reactions which compromises the clarity of the representation. Also, it is often convenient to have in the same view some specific pathways in more detail while the others, which are not of interest at the moment, are kept closed.

\subsubsection{Retrieving hypothetical neighbors}

As described previously, the links between reactions and pathways are given by Reactome through either regulation or precedingEvent. However, there could also be a link from a reaction that produces a metabolite to a reaction in the same compartment that consumes that metabolite. Since Reactome includes this compartment information, we can also draw edges between reactions that share substrates or products. This option (Get hypothetical neighbors) is also in the context menu (Fig. 1) and may be used for more than one reaction at a time by selecting multiple reactions. If the reactions that could be influenced by the given reaction(s) are already on screen, only a black dashed arrow is displayed between them. If the reaction is not already on the screen it is added, before adding the black dashed arrow. The reactions, that share compounds with the given reactions, are called "hypothetical neighbors". We call these "hypothetical" because Reactome has decided not to show these edges, although the edges are in theory possible.

The edges which are regularly represented in ReConn are the so called nextEvent edges, inserted by the curators. These edges overlap with the usual "main stream" of metabolites, whereas in fact in many diseases the normal sate is perturbed as a result of which alternative paths can become important. With the feature of retrieving hypothetical neighbors one can add alternative reactions where in principle also metabolites can be consumed. By representing the differential expression one can check if indeed an alternative path is used. 


\section{Comparison of Currently Available Tools}

In this section we compare several well known interface and visualization tools, including ReConn. To this end we first give a set of features the tools should provide. After a brief overview of the tools, we compare them and discuss the results.

\subsection{General requirements}

We identify a set of useful features of the interface and visualization tools which are important for their applicability. Taking into account the multilevel and multiscale approach to deal with biological networks, as well as the abundance of data sources, we distinguish three different aspects:

- Single level: Visual analysis may become difficult for large networks. One solution to this problem is to make networks smaller, by either aggregating or selecting network elements. This reduces the number of visual items, making the analysis and interpretation of the network simpler.

Further, it might help if the networks are represented in a familiar way, like they appear in textbook figures. For example, the TCA cycle is preferably visualized as a circle-like structure.

Also the network appearance can be customized using interactive visualization. This means that one should have the possibility to select parts of the biological system that are to be visible at a given time. The visualization tool should ideally also offer facilities to add related entities and consequently to derive other networks. Moreover, it should be possible to remove network elements. All these options are aimed to focus on the interesting parts of the network. When network elements are selected, information of the relations between visible elements and the hidden ones should still remain accessible.

Thus, we can distill the following requirements for inspecting biological networks at a single level:

- Selective or aggregated display of network elements,

- Interactive graph representation,

- Textbook-like layout,

- Network selection,

- Show relations between hidden and visible elements.

- Multilevel: When integrating data from, for example, microarray experiments and protein networks, we are interested in more than one level. Apart from the above mentioned requirements, for each individual level additional functionality is needed. Data from multiple levels should be integrated into a single network that represents the entire system. Because data from different levels of the system usually have different names or identifiers, software should help the user to integrate this information. Therefore, an extra requirement with regard to multiple levels is:

- Integrate data from multiple levels into a single network. 
- Multiple sources: When networks are available from multiple sources (e.g. different databases or different species), tools should facilitate the identification of common parts and parts that are different. In short, the additional requirements for multiple sources are:

- Show networks from different sources,

- Compare networks from different sources.

\subsection{Tools}

GenMAPP ${ }^{1}$ is one of the most widely used tools to visualize genomics data obtained from microarrays on pathways. Pathways are considered as groups of genes called MAPPs. GenMAPP offers two possibilities for inspection: one can consider a single pathway and show the genes that are up- or down-regulated, or one can get a sorted list of pathways that are most affected.

$\mathrm{KEGG}^{2}$ is a well known pathway database that has information on various levels including proteins, genes and metabolites. KEGG also has tools for drawing chemical structures (KegDraw) and for the analysis of transcriptome data (KegArray).

Pathway Tools ${ }^{3}$ is a software package to visualize and to create pathway/ genome databases (PGDBs). The creation of species specific PGDBs is an important feature. However, with respect to the requirements, only the visual representation of the pathways and the visualization of omics data on those pathways are considered.

$\mathrm{BiNoM}^{4}$ is a Cytoscape plug-in for visualizing and analyzing BioPAX, SBML and CellDesigner files. BiNoM supports three types of different visualizations: reaction graphs, protein-protein interaction graphs, and pathway hierarchy graphs.

BioNetBuilder ${ }^{5}$ has been developed to provide a more user friendly interface to create biological networks from several databases. These databases include: BIND, ${ }^{7}$ BioGRID,${ }^{8}$ DIP,${ }^{9}$ GO,${ }^{10}$ HPRD,${ }^{11}$ KEGG and Prolinks. ${ }^{12}$

\subsection{Comparison}

Below we give an overview of each of the features and discuss by which tools and to which extent it has been implemented.

\subsubsection{Selective or aggregated display of network elements}

GenMAPP's main visual elements are boxes that represent genes and in addition only the main metabolites are depicted. KEGG has a similar approach with the difference that enzymes instead of genes are used as main visual elements. In Pathway tools the nodes in the graphs represent metabolites and the edges represent reactions whereas genes and enzymes are omitted. Graphs created by Pathway tools usually contain more nodes and edges than both KEGG and GenMAPP. In BiNoM and BioNetBuilder by default all elements from the network are depicted, however, using basic Cytoscape functionality elements can be selected and hidden. 


\subsubsection{Interactive graph representation}

To some extent GenMAPP and BiNoM have an interactive graph representation. However, when nodes are removed from a view, information about the connectivity to other nodes is lost. The interactive graph representation of BioNetBuilder is similar to that of BiNoM, because they both use Cytoscape for the visualization. In Pathway Tools one has a very limited interactivity. For instance one can zoom in and out, but not more than that. KEGG has no interactive network representations.

\subsubsection{Textbook-like layout}

The MAPPs that can be downloaded from GenMAPP have a layout that resembles textbook figures. Similarly, in KEGG and Pathway Tools the pathways have been created with a textbook-like layout. Since there is no layout information in either BioPAX or SBML, BiNoM cannot create a layout that resembles a text-book one. A layout in BioNetBuilder is automatically generated, hence not necessarily resembling that of textbook images.

\subsubsection{Network selection and modification}

GenMAPP comes with predefined networks that do not allow for selection within a pathway, although one can create new MAPPs manually. KEGG layouts cannot be altered or selected from. Also Pathway Tools layout cannot be changed and network selection is not possible from the visualizations of the PGDBs. Although it is possible to create new PGDBs for specific purposes, this is not an easy task.

In BiNoM, apart from the Cytoscape features for selecting interesting parts of the network, some of additional data analysis features are provided. In BioNetBuilder network selection is only possible by using Cytoscape features.

\subsubsection{Show relations between hidden and visible elements}

In GenMAPP the relationships between the different MAPPs are shown as hyperlinks. Hyperlinks are also used in KEGG and Pathway Tools to represent the relationship between different pathways. In BiNoM and BioNetBuilder the relationship between hidden and visible elements is not shown.

\subsubsection{Integrating data from multiple levels into a single network}

GenMAPP only supports genes, therefore the multilevel requirements are not met. KegArray allows visualization of just a combination of transcriptomics and metabolomics data. The Pathway Tools Omics Viewer, as its name suggests, is capable to integrate data from multiple levels into one network. Networks from different databases can be generated by BioNetBuilder, each of them being related to one level of the system.

In KEGG the user is restricted to the identifiers that are defined by the KEGG database. For example, Affymetrix identifiers are not supported. Therefore the 
integration property is not satisfied. BiNoM can show different levels in one network. The user has to link the data to the visual elements, using Cytoscapes functionality to import expression data, by manually coupling identifiers used in the data to the visual elements. Adding identifiers to the network facilitates the expression data import functionality of Cytoscape.

\subsubsection{Show networks from different sources}

GenMAPP supports custom MAPPs, which can be shared with others. KEGG does not support the visualization or comparison of pathways from other databases. Thus, none of the multiple sources requirement is satisfied. In Pathway Tools all PGDBs are derivatives from one database called MetaCyc, which contains only metabolic pathways. The Pathway Tools Omics Viewer has therefore no options to show or compare networks from different sources. BiNoM can display networks from different sources. Since BioPAX and SBML are specifically designed to exchange pathway information, many databases support these file formats. In BioNetBuilder different networks can be combined into one view. Color coding is used to specify from which database the interaction was derived.

\subsubsection{Compare networks}

Although GenMAPP supports shared custom maps, it has no options for comparing different MAPPs with each other. As mentioned above both KEGG and Pathway Tools do not satisfy any of the multiple sources requirements. BiNoM is capable

Table 1. Comparison of different software in relation to the requirements.

\begin{tabular}{|c|c|c|c|c|c|c|}
\hline Requirement & GenMAPP & KEGG & Pathway Tools & BiNoM & BioNetBuilder & ReConn \\
\hline \multicolumn{7}{|l|}{ Single level } \\
\hline $\begin{array}{l}\text { Selective or aggregated } \\
\text { display }\end{array}$ & + & + & + & $+/-$ & $+/-$ & + \\
\hline $\begin{array}{l}\text { Interactive graph } \\
\text { representation }\end{array}$ & $+/-$ & - & $+/-$ & + & + & + \\
\hline Textbook-like layout & + & + & + & - & - & + \\
\hline Network selection & - & - & - & + & + & + \\
\hline $\begin{array}{l}\text { Relations hidden and } \\
\text { visible parts }\end{array}$ & $+/-$ & $+1-$ & $+/-$ & - & - & + \\
\hline \multicolumn{7}{|l|}{ Multilevel } \\
\hline $\begin{array}{l}\text { Data from different } \\
\text { levels of the system }\end{array}$ & - & $+/-$ & + & + & $+/-$ & + \\
\hline $\begin{array}{l}\text { Data from multiple } \\
\text { levels into one } \\
\text { network }\end{array}$ & - & - & + & $+/-$ & $+/-$ & + \\
\hline \multicolumn{7}{|l|}{ Multiple sources } \\
\hline $\begin{array}{c}\text { Show networks from } \\
\text { different sources }\end{array}$ & $+1-$ & - & - & + & + & - \\
\hline $\begin{array}{l}\text { Compare networks } \\
\text { from different } \\
\text { sources }\end{array}$ & - & - & - & + & + & - \\
\hline
\end{tabular}


of comparing networks, by visualizing the differences between two networks. In BioNetBuilder additional color coding indicates when an interaction has been found in multiple databases.

In Table 1 the comparison of the different software packages is summarized. The functionalities of ReConn with respect to the requirements are shown in the last column. It follows from the table that none of the software packages fulfills all requirements to a full extent. However, one can also argue that, according to these requirements, ReConn is the most complete tool. ReConn covers the whole requirement set, apart from the multiple source aspect requirements. Since there are existing specialized tools that satisfactory meet those requirements, we decided not to include them in ReConn.

\section{Implementation Details}

\subsection{Visualization}

ReConn is implemented as a Cytoscape plug-in. Cytoscape was chosen for a number of reasons. Firstly, Cytoscape allows for focusing on the functionality instead of writing another graph visualization program. A second advantage is that Cytoscape is open source and written in the Java ${ }^{18}$ programming language. This has resulted in an active community of developers and a community-driven design. This is important for the longevity of the project and for many possible users of the application. Also it means that further innovation is to be expected and that there is support for both users and developers. Moreover, the well-documented API of Cytoscape allows for easy plug-in development. The developer can either make use of high level function calls and produce results quickly, or dive into the core code and tune the behavior of Cytoscape for a specific case.

\subsection{Data connection}

Although Reactome offers a $\mathrm{SOAP}^{19} \mathrm{API}^{20}$ to access the Reactome data, we opted for a direct $\mathrm{MySQL}^{21}$ database connection for reasons of speed. Using MySQL, we were able to write queries that collect data in a reasonable time frame, e.g. displaying all human reactions takes less than twenty seconds on a standard PC, see Table 2. For some larger pathways, or the overview containing all the reactions,

Table 2. Computation times for the more time consuming actions in ReConn.

\begin{tabular}{ll}
\hline Action & Computation time \\
\hline Show all human reactions & 19 seconds \\
Map 54675 expression values on all human reactions & 22 seconds \\
PathwayFinder on 54675 expression values & 22 seconds \\
\hline
\end{tabular}

Note: Computation times for the more time consuming actions in ReConn. These values were obtained with a Pentium $43.0 \mathrm{GHz}$ computer with $1024 \mathrm{MB}$ RAM and a high-speed Internet connection $(4 \mathrm{Mb} / \mathrm{s})$. 
this performance would not have been possible using the SOAP API. The overview of all reactions took over half an hour when using the SOAP API. Reactome does not host a publicly accessible MySQL server, therefore we are hosting it on one of our own servers. We will update the database within a month after each Reactome release, allowing us to adapt the SQL queries in case Reactome changes the database schema. Another advantage of directly connecting to a MySQL instance of the Reactome database is the freedom gained in writing our own queries. All interactions with the database are kept inside a separate DatabaseConnect class. The information derived from the database is stored in our own data structures.

\section{Discussion and Conclusions}

We have developed ReConn to provide novel flexible representations of biological networks. With ReConn new pathways can be derived using the features "Grow pathway from compound" or the "Get hypothetical neighbor" options. Another specific feature, calculating alternative routes between network elements, can potentially facilitate the design and analysis of experiments in which parts of the network are disabled, e.g. knockout experiments.

Since we wanted ReConn to be a fully-fledged tool we aimed in covering as much as possible the useful capabilities offered by the other tools. We first defined a set of requirements that are essential for this kind of tools. Considering the multilevel structure of biological systems, we distinguish three different aspects of network visualization: single level, multilevel, and multiple sources. Apart from the multiple source aspect requirements, ReConn covers the complete requirement set. The former have not been implemented, because there are existing tools that meet those requirements. With respect to the single level requirements, ReConn combines information into a graph that has reactions as nodes, relations between reactions as edges, and visualizes this graph interactively. When these networks are loaded, the layout resembles figures from textbooks. ReConn allows network selection through growing of networks and adding "hypothetical neighbors". Relations between visible and hidden nodes can be retrieved using the "hypothetical neighbors" feature.

Concerning the multilevel requirements, ReConn displays information about multiple levels of the system in one view. Another feature of ReConn is the mapping of experimental data onto the networks. Currently available programs that are capable of this do not meet the other above mentioned requirements to a full extent.

Although it has been developed as an interface for Reactome, ReConn can be adapted in a fairly straightforward way to work with other databases.

\section{Availability and Requirements}

Home page: http://bmi.bmt.tue.nl/reconn/

Code availability: http://sourceforge.net/projects/reconnplugin/

Operating system: Platform independent 
Programming language: Java

Other requirements: Java 1.4 or higher, Cytoscape 2.6 or higher

License: GPLv2

\section{Acknowledgments}

The authors would like to thank the Cytoscape development team for their technical support. WL and DB are supported by the BioRange program of The Netherlands Bioinformatics Centre (NBIC), which is supported by a BSIK grant through The Netherlands Genomics Initiative (NGI).

\section{References}

1. Dahlquist KD, Salomonis N, Vranizan K, Lawlor SC, Conklin BR, GenMAPP, a new tool for viewing and analyzing microarray data on biological pathways, Nat Genet 31:19-20, 2002.

2. Kanehisa M, Goto S, Kawashima S, Nakaya A, The KEGG databases at GenomeNet, Nucleic Acids Res 30(1):42-46, 2002.

3. Paley SM, Karp PD, The pathway tools cellular overview diagram and omics viewer, Nucleic Acids Res 34(13):3771, 2006.

4. Zinovyev A, Viara E, Calzone L, Barillot E, BiNoM: A cytoscape plugin for manipulating and analyzing biological networks, Bioinformatics 24(6):876, 2008.

5. Avila-Campillo I, Drew K, Lin J, Reiss DJ, Bonneau R, BioNetBuilder: Automatic integration of biological networks, Bioinformatics 23(3):392, 2007.

6. Joshi-Tope G et al. , Reactome: A knowledgebase of biological pathways, Nucleic Acids Res 33:D428-D432, 2005.

7. Bader GD, Donaldson I, Wolting C, Ouellette BF, Pawson T, Hogue CWV, BIND-the biomolecular interaction network database, Nucleic Acids Res 29(1):242, 2001.

8. Stark C, Breitkreutz BJ, Reguly T, Boucher L, Breitkreutz A, Tyers M, BioGRID: A general repository for interaction datasets, Nucleic Acids Res 34(Database Issue): D535, 2006.

9. Salwinski L, Miller CS, Smith AJ, Pettit FK, Bowie JU, Eisenberg D, The database of interacting proteins: 2004 update, Nucleic Acids Res 32(Database Issue):D449, 2004.

10. Ashburner $\mathrm{M}$ et al., Gene Ontology: Tool for the unification of biology, Nat Genet 25(1):25-29, 2000.

11. Peri S et al., Development of human protein reference database as an initial platform for approaching systems biology in humans, Genome Res 13(10):2363-2371, 2003.

12. Bowers P, Pellegrini M, Thompson M, Fierro J, Yeates T, Eisenberg D, Prolinks: A database of protein functional linkages derived from coevolution, Genome Bio 5(5): R35, 2004.

13. Lockhart DJ, Dong H, Byrne MC, Follettie MT, Gallo MV, Chee MS, Mittmann M, Wang C, Kobayashi M, Horton H, Brown EL, Expression monitoring by hybridization to high-density oligonucleotide arrays, Nat Biotechnol 14:1675-1680, 1996.

14. Velculescu VE, Zhang L, Vogelstein B, Kinzler KW, Serial analysis of gene expression, Science 270(5235):484, 1995.

15. Burnette WN, Western Blotting: Electrophoretic transfer of proteins from sodium dodecyl sulfate-polyacrylamide gels to unmodified nitrocellulose and radiographic detection with antibody and radioiodinated protein A, Anal Biochem 112(2):195-203, 1981. 
16. Aebersold R, Mann M, Mass spectrometry-based proteomics, Nature 422(6928):198-207, 2003.

17. Shannon P, Markiel A, Ozier O, Baliga NS, Wang JT, Ramage D, Amin N, Schwikowski B, Ideker T, Cytoscape: A software environment for integrated models of biomolecular interaction networks, Genome Res 13(11):2498, 2003.

18. JAVA. Available at http://java.sun.com/. Accessed 12 December 2012.

19. SOAP. Available at http://www.w3.org/TR/soap/. Accessed 12 December 2012.

20. Reactome SOAP. Available at http://www.reactome.org:8080/caBIOWebApp/services/ caBIOService?wsdl. Accessed 12 December 2012.

21. MySQL. Available at http://www.mysql.com/. Accessed 12 December 2012.

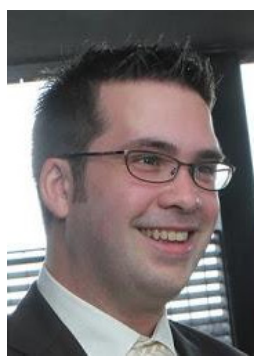

Willem Ligtenberg received his M.Sc. degree in Biomedical Engineering from Eindhoven University of Technology, the Netherlands in 2006. As a part of this study, he spent four months at the Bioinformatics Research Centre at Glasgow University (Scotland). After obtaining his M.Sc. degree, he started his $\mathrm{PhD}$ research in the BioModeling and BioInformatics group of the department of Biomedical Engineering at the Eindhoven University of Technology. In this research, he studied how network theoretical approaches can be applied on biological networks.

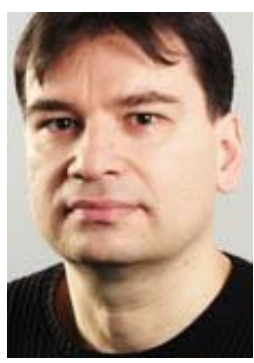

Dragan Bošnački received his B.Sc. degree in electrical enginering (Faculty of Electrical Engineering) and M.Sc. degree in computer science (Institute of Informatics, Faculty of Natural Sciences) from the St. Cyril and Methodius University in Skopje, Macedonia, and a Ph.D. in computer science, from Eindhoven University of Technology, in The Netherlands. Currently he is an assistant professor at the Biomedical Engineering Department of Eindhoven University of Technology. His main research interests are in Bioinformatics and formal methods. 


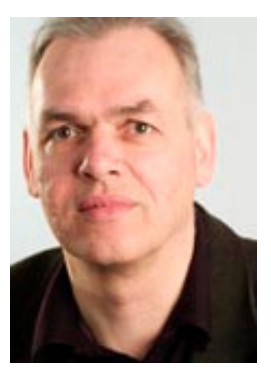

Peter Hilbers is fulltime professor "BioModeling and BioInformatics" at the biomedical engineering department of the TU Eindhoven. He studied Mathematics at the University of Groningen, where he also completed his Ph.D. study on "Mapping of algorithms on processor networks" in 1989. From 1989 till 1996, he worked at the Shell Laboratories in Amsterdam. He was appointed as a part-time Professor "Large scale computing" of the mathematics and computer science department at the Eindhoven University in 1993, and as a full-time Professor in 1996. Since 2005, he is involved in the research and edcucational programs of the Sino-Dutch Biomedical \& Information Engineering School of NorthEastern University, Shenyang, China. In October 2007, he became the dean of the department of BioMedical Engineering of the TU Eindhoven. 